Archived version from NCDOCKS Institutional Repository http://libres.uncg.edu/ir/asu/

\title{
Appalachľan
}

B O O N , N O R T H C A R O L I N A

\section{Voluntary Sleep Choice And Its Effects On Bayesian Decisions}

\author{
By: David L. Dickinson, Sean P.A. Drummond, \& Jeff Dyche
}

\begin{abstract}
This study examines whether voluntary sleep restriction at commonly experienced levels impacts decision making in a Bayesian choice task. Participants recruited were largely traditional age college students from a regional state university $(\mathrm{n}=100)$ and a federal military academy $(\mathrm{n}=99 ; \mathrm{n}=56$ and 43 , respectively, used in final analysis). Sleep was measured by actigraphy over a one-week period, followed by performance of a decision task. The task involved two sources of information, base rate odds and sample evidence, with subjects asked to make a probability judgment. Results found that subjects with nightly sleep $<6 \mathrm{hr}$ (sleep deprived $=\mathrm{SD}$ ), relative to those with $>7 \mathrm{hr}$, placed less decision weight on new evidence, relative to base rate information, in making difficult choices. This result is strongest among female subjects. For easier choices, voluntary SD did not affect relative decision weights placed on the two sources of available information.
\end{abstract}

Dickinson, D. L., et al. (2016). "Voluntary Sleep Choice and Its Effects on Bayesian Decisions." Behavioral Sleep Medicine 14(5): 501-513. https://doi.org/10.1080/15402002.2015.1028064. Publisher version of record available at: https://www.tandfonline.com/doi/full/10.1080/15402002.2015.1028064 
Voluntary sleep choice and its effects on Bayesian decisions: A study of two samples of college students.

\author{
David L. Dickinson, $\mathrm{PhD}^{1}$ \\ Sean P.A. Drummond, $\mathrm{PhD}^{2}$ \\ Jeff Dyche, $\mathrm{PhD}^{3}$
}

1. Appalachian State University, Department of Economics

2. VA San Diego Healthcare System, Psychology Service; University of California San Diego, Department of Psychiatry; VA San Diego Center of Excellence in Stress and Mental Health

3. James Madison University, Department of Psychology

Corresponding Author:

Sean P.A. Drummond, PhD

VA San Diego Healthcare System

3350 La Jolla Village Dr., MC 116B

San Diego, CA, 92161

8586421274 (phone)

drummond@ucsd.edu (email)

All authors declare they have no conflicts of interest.

Number of words: 4999

Number of references: 23

AUTHOR CONTRIBUTIONS:

Study design (DLD, SPAD), data acquisition (DLD, SPAD, JD), data analysis (DLD, SPAD), interpretation of data (DLD, SPAD) manuscript preparation (DLD, SPAD, JD). 


\begin{abstract}
This study examines whether voluntary sleep restriction at commonly experienced levels impacts decision-making in a Bayesian choice task. Participants were largely traditional age college students from a regional state university $(\mathrm{n}=100)$ and a federal military academy $(\mathrm{n}=99)$. Sleep was measured by actigraphy over a one-week period, followed by performance of a decision task. The task involved two sources of information, base rate odds and sample evidence, with subjects asked to make a probability judgment. Results found that subjects with nightly sleep $<6$ $\mathrm{hr}$ (sleep deprived=SD), relative to those with $>7 \mathrm{hr}$, placed less decision weight on new evidence, relative to base rate information, in making difficult choices. This result is mediated by gender and differs when comparing cadets with traditional student subjects. For easier choices, voluntary SD did not affect relative decision weights placed on the two sources of available information, and results were similar across institutions and by gender.
\end{abstract}




\section{Introduction}

The average U.S. adult now sleeps less than seven hours per night on weekdays, and approximately one in six adults sleep less than 6 hours per night (NSF, 2012). This leads to health concerns regarding insufficient sleep, but an even more serious worry may be the negative spillover effect one's sleepiness can have on others. Occupations implicated in producing sleepdeprived workers are often those where public health is at risk in the event of decision errors. Emergency service/safety workers, long-haul truckers, air traffic controllers, doctors, and nurses are more likely than average to perform shift work (McMenamin, 2007) and/or report insufficient sleep (NSF, 2012).

Laboratory sleep deprivation (lab SD) protocols have been used to study the impact of sleep deprivation on decision under risk or uncertainty, which appear sensitive to sleep loss (Harrison and Horne, 2000). In some instances, lab SD demonstrates an increased preference for or desensitization to risk (Killgore et al., 2012; McKenna et al., 2007; Venkatraman et al., 2011), and neural evidence indicates risk-modulating receptors are down-regulated following lab SD (Volkow et al., 2012). However, other studies show no impact of lab SD on risk preference (Venkatraman et al., 2011), or even a decrease in risk taking following sleep loss (Acheson et al. 2007; Chaumet et al., 2009). Different results are likely due to protocol differences (i.e., type of sleep loss studied), or task-specific details regarding objective risk versus risk with missing information (McKenna et al., 2007).

An understanding of how SD impacts the use of available information is critical towards understanding SD effects on risky choice (Dickinson and Drummond, 2008). The use of both prior information (e.g., base rate odds) and newly acquired evidence in formulating subjective beliefs regarding an event is referred to as Bayesian updating or inference-Bayes' rule calculates the updated probability that results from combining base rates and new evidence. An 
investigation of Bayesian updating is therefore fundamental towards understanding decision making in probabilistic environments. In our previous study (Dickinson and Drummond, 2008), we found that 23 hours of lab TSD decreased estimated decision weights placed on new information when making choices.

This paper expands our prior work with experimental TSD to shed light on how voluntary sleep restriction impacts Bayesian decisions. Here, we focus on an ecologically valid environment of voluntary sleep choice. While lacking laboratory control, the trade-off is that voluntary sleep choice is more directly applicable to real world decision makers. Some have argued that naturalistic setting studies may be undervalued in randomized trial research (Blacker and Moritmore, 1996), especially when important environmental (e.g., social) factors go hand-inhand with real world “dosage” choices of key variables such as sleep choice or alcohol consumption (Lyvers and Tobias-Webb, 2010; Scholey et al., 2012).

We used actigraphy to monitor naturally occurring sleep in two samples, while exercising control over circadian influences during testing. One sample is from a regional public university, while the other is from a national service academy. Following one week of actigraphymonitored sleep, subjects took a computerized version of the previously studied Bayesian task (Dickinson and Drummond, 2008), with our aim being to study whether lab TSD effects are transferable to more ecologically valid environments of voluntary sleep restriction. We additionally examined gender as a mediating factor in the data analysis. Studies in the sleep literature have generally found sleep deprivation effects on cognitive performance do not vary by gender among civilians (Frey et al., 2004) or in military settings (Chelette et al., 1998). However, when looking at tasks very similar to ours, both Lundborg and Andersson (2008) and Cameron (2005) find gender specific effects regarding the use of new information in making risk 
assessment. Given these differential predictions regarding gender effects, based on the sleep and decision literatures, we wanted examine whether gender may mediate voluntary sleep restriction effects on decision making in the specific domain of Bayesian choice.

\section{Methods}

The studies were approved by the Institutional Review Boards of Appalachian State University and the U.S. Air Force Academy, and subjects were compensated for their participation.

\section{Subjects and Procedures}

Recruitment procedures were similar at both Appalachian State University (ASU, n=100, 50

female, age=22.98 \pm 7.98 yrs old (age range 18-57)) and the U.S. Air Force Academy (USAFA, $\mathrm{n}=99$, 48 female, age=20.3 \pm 1.63 yrs old (age range $17-25)$ ). Subjects were recruited to take part in a study that involved completing sleep diaries and wearing an actigraphy device for one week (Actiwatch-64, Phillips Respironics at ASU; Ambulatory Monitoring, Inc, Motionlogger at USAFA), and then being administered a set of decision tasks at the end of that week. In addition to the Bayesian decision task reported here, we administered two other decision tasks in counterbalanced order during the same testing session (results will not be presented here).

Actigraphy malfunctions and subject withdrawal eliminated 19 subjects from USAFA (no such instances in the ASU data), reducing the USAFA sample to 80 subjects. For the analyses, we also excluded subjects with objectively-scored average nightly sleep between 6-7 hours because, while some studies define chronic partial sleep deprivation as restricting subjects to 7 hours in bed/night (Van Dongen, et al., 2003), epidemiological surveys have reported typical average nightly sleep duration in the 6-7 hour range (NSF, 2012). Thus, it was not clear if sleep 
in that range should be considered SD or normally rested. This exclusion reduced the final sample size to 56 (ASU) and 53 (USAFA) subjects, but it eliminated difficult-to-score sleep durations that would have added noise to the data.

Control for circadian influences was slightly different at ASU and USAFA. Subjects at USAFA were likely a mix of diurnal preference types because chronotype data were not collected. Sessions at USAFA took place during a common block of time (i.e., 11:00 a.m. to 1:30 p.m.) intended to avoid conflicts with morning or evening-type wakefulness troughs. ASU Subjects were recruited from a database of subjects who had been administered a validated reduced-form version of the Horne and Östberg (1976) morningness-eveningness questionnaire (Adan and Almirall, 1991). Only "intermediate-type” subjects were recruited for this experiment, which took place at a non-extreme times (11:00 a.m.-3:30 p.m.).

\section{Sleep Protocol and Actigraphy}

Subjects reported to their respective experiment lab (ASU or USAFA) for an initial session that included informed consent, actigraphy fitting and instructions, and sleep diary information (used to complement scoring of actigraphy data following validated actigraphy methods). Subjects were instructed to wear the actigraphy 24/7 except for exceptional circumstances (e.g., playing contact sports, harsh chemical exposure, extended water exposure, etc). They reported back to the lab one week later, returned the actigraphy device, sleep diaries, and were administered the decision tasks, which included the Bayes task. Subjects were paid in cash at the end of the decision experiment session. ASU subjects received a $\$ 30$ fixed payment for providing the actigraphy data, and variable payments for outcomes in the set of decision tasks averaged approximately $\$ 21$ additional (min \$0, max \$69.25). USAFA subjects received no monetary fixed payment, some (but not all) were given extra credit for participation, and 
variables payments for all tasks averaged $\$ 37.65$ (min $\$ 0$, max $\$ 225.00)$. The differences in base rate payments relate to different IRB regulations at the two institutions. As noted in the next section, each subject received either \$2 or \$12 specifically for the task reported here.

\section{The Bayes’ Rule Task}

The task administered was a computerized version of the task used in our earlier study with a lab TSD protocol (Dickinson and Drummond, 2008), which modified an existing design (Grether, 1980). Subjects were presented with an unknown environment (see Fig. 1), and told that balls would be drawn with replacement from either the LEFT or RIGHT box. Their task was to indicate the box from which the balls were drawn. Both boxes were populated with black and white balls; the LEFT box contained two black and one white ball, while the RIGHT box contained two white and one black ball. Subjects were informed of base rate odds directly beneath each box. These base rates were used to select a box, but the subjects were not shown the selection result. Rather, the selected box was then used to draw five balls with replacement, and the resultant sample draw (i.e., the new information) was shown at the bottom of the stimulus. Thus, the subjects had two available sources of information—base rate probabilities and sample evidence—-that could be used to make one's assessment of the box utilized.

In each trial, subjects had 6 seconds to indicate which box they felt the balls came from, and then a new stimulus would appear. Stimuli differed by base rate odds and sample evidence. Each subject saw a total of 48 task stimuli or trials; 16 of these checked comprehension by using base rate odds of 0/6 for one of the boxes. The remaining 32 stimuli can be divided into 16 "Easy" and 16 "Hard” trials. We considered an Easy trail to be one where the posterior probability of the more likely box, which can be calculated by Bayes' rule, to be $\geq 80 \%$. If the Bayesian odds of the more likely box were between $50 \%-80 \%$, we considered this a Hard trial 
because the posterior odds were not so extreme in favor of the more likely box. The task was incentivized as follows: subjects were told that one trial would be selected at random and their choice of box used in that trial would result in a payoff of \$12 if correct, but $\$ 2$ if incorrect. Subjects were given no feedback on accuracy or outcomes of any trial until the end of the experiment when the payoff trial was drawn at random.

\section{Bayes’ decision model estimation}

The task allowed us to estimate the decision weight subjects place on each of the two information sources present in the task: the base rate (initial) information, and the sample draw (new) information. Following Grether (1980), the basic decision model estimated is:

$$
Y_{i t}^{*}=\alpha+\beta_{1} \ln L R(L)_{t}+\beta_{2} \ln \left(\frac{P_{L}}{1-P_{L}}\right)_{t}+\mu_{i}+\epsilon_{i t}
$$

where $Y_{i t}^{*}$ is the subjective log odds in favor of the LEFT box in trial $t$ for subject $i$, which is a function of both evidence and base rate information in favor of the LEFT box for that trial. Specifically, $\ln L R(L)_{t}$ is the $\log$ of the statistical likelihood ratio of the LEFT box (i.e., the evidence variable), which is defined as the likelihood of observing the sample evidence if the balls were drawn from the LEFT box divided by the likelihood of observing that sample if the balls were drawn from the RIGHT box. The other variable, $\ln \left(\frac{P_{L}}{1-P_{L}}\right)_{t}$, is the log of the base rate odds ratio for the LEFT box (i.e., the base rate variable). Henceforth, we shall refer to the variables $\ln L R(L)$ and $\ln \left(\frac{P_{L}}{1-P_{L}}\right)_{t}$ as "Evidence" and "Base Rate", respectively. Because $Y_{i t}^{*}$ is not observed, the model was estimated using probit techniques, where our observable dependent variable $Y_{i t}$ was set equal to one if $Y_{i t}^{*} \geq 0$. Grether (1980) indicates that a Bayesian subject should place equal weight on both sources of information in forming one's belief. 
To the base model (1) we added a dichotomous variable for sleep deprivation (SD) to identify subjects averaging $<6 \mathrm{hr}$ sleep/night as sleep deprived (SD=1) versus those who averaged $>7 \mathrm{hr}$ sleep/night (normally-rested, NR) (i.e., SD=0). We also added variables to capture the interaction of SD with each of the two sources of information. Our results are qualitatively similar if retaining sleep quantity as a continuous variable, but we report results based on the dichotomous SD for simplicity. Similarly, results were qualitatively similar defining SD as nightly sleep $<6.5$ hrs and normally rested as nightly sleep $>6.5$ hrs. We analyzed gender effects using separate estimations for male and female subsamples in both Easy (Table 1) and Hard trials (Table 2). An alternative approach is to pool the male and female data and include gender and interactions in the estimations, but this requires triple-interaction terms that are difficult to interpret. We do not report results of such interactions here, but they are consistent with what we report using separate estimations for male and female subjects (and available on request). We favored separating the estimations by gender for ease of exposition.

\section{Results}

Figure 2 shows a smoothed histogram estimate of average nightly sleep quantities over the actigraphy phase of our study for the ASU $(n=100)$ and USAFA $(n=80)$ subjects who completed the study and provided valid actigraph data. This includes subjects sleeping 6-7/hrs per night, who we drop from the decision analysis as noted previously. Average nightly sleep was significantly greater in the USAFA sample compared to the ASU sample but, in general, these sleep quantities corresponded closely to recent survey reports of U.S. adult sleep habits (NSF, 2012). Because of sleep duration differences, the clear distinction between type of institution, and some of the aforementioned differences in protocol, we analyzed the data 
separately for each institution. For each institution's data set, we show estimation results when splitting the sample by gender as well when pooling across gender.

The Easy trial results (Table 1) are as follows: Subjects weight both sources of information in making choices, but most subsamples showed more decision weight placed on base rate information (i.e., subjects overweight the odds, relative to the evidence, on Easy trials). This tendency was statistically significant for all models $(\mathrm{p}<.01)$ except ASU-male subjects (p>.10: all tests were $X^{2}$ tests of coefficient equality). Voluntary sleep restriction did not significantly change the relative decision weighting for any of the subsamples at either institution ( $>$.10 for all cases).

On Hard trials (Table 2), normally-rested ASU subjects significantly overweighted Evidence ( $<.01$ for both male and female samples), while normally-rested USAFA subjects placed statistically identical weight on Base Rate and Evidence. A common theme emerged when examining the effect of voluntary sleep restriction on Hard trials. Here, except for USAFA males, the model estimates indicated a significant shift of relative decision weight away from evidence and towards the base rates.

To help visualize these results, see Figures 3-4. Because others have cautioned against comparing coefficients across non-linear probit models (Allison, 1999; Williams, 2009), we created normalized decision weights for these figures. The "relative decision weight” metric expresses the difference in the Evidence coefficient, $\beta_{1}$, and Base Rate coefficient, $\beta_{2}$, over to the sum of those same estimated coefficient weights for a given model: $\frac{\beta_{1}-\beta_{2}}{\beta_{1}+\beta_{2}}$. This created a standardized relative decision weight $\in[-1,+1]$. Positive values on this scale indicate overweighting the evidence, negative values indicate overweighting the base rates, and a value of zero indicates equal weighting of the two information sources. The test of $\beta_{1}=\beta_{2}$ for model (1) 
has been used as a criterion for Bayesian choice (Grether, 1980), so for simplicity we considered $\beta_{1}>\beta_{2}$ or $\beta_{1}<\beta_{2}$ as over-weighting of one source of information or the other. Figure 3 shows the general over-weighting of Base Rate on Easy trails, regardless of whether subjects were SD or normally-rested (NR) (significant for everyone but ASU males). Figure 4 shows that, for Hard trials, SD shifts estimated decision weights towards Base Rate for everyone except USAFA males. Figure 4 also highlights that on Hard trials females tend towards a relatively higher decision weight on base rates compared to males, especially for USAFA females who are voluntarily sleep restricted.

Thus, SD only affected decision weights during the difficult—perhaps more cognitively challenging - Hard trials. The result was found in 3 of the 4 subsamples. These decision model differences notwithstanding, we found that SD did not significantly affect subject accuracy (i.e., likelihood they chose the Bayesian-more-likely box). Only among ASU males on Hard trials did we find some evidence that voluntary SD reduced Bayesian accuracy $(\mathrm{p}<.05$ using a regression of accuracy $(=0,1)$ as the dependent variable, and SD as the only independent variable. For the other three subsamples on Hard trials and for all subsamples in Easy trials, the SD coefficient was statistically insignificant).

\section{Discussion}

This study used voluntary, but objectively measured, sleep duration to study sleep restriction effects on a fundamental decision process. We extended our previous research (Dickinson and Drummond, 2008) by using a naturalistic setting with two separate university samples and self-selected sleep schedules. This is the first study to examine a standardized decision task in the context of naturally occurring sleep durations and in such large samples. 
Our results showed that, in general, when the total available information strongly favors one decision (i.e., Easy choice), subjects tend to weight the base rate information (i.e., the odds) relatively more than the sample evidence. In general, this may be because the base rate information was more readily usable. Evidence information in our stimulus had to go through an extra step of conversion from visual information to probability. We estimated no significant effect of voluntary SD for Easy trials.

As for Hard choices, there was a tendency to place relatively more decision weight on evidence information when normally rested, but this was only present in the ASU subjects. However, voluntary sleep restriction was also associated with significant decision weight effects on Hard trials. For all subjects except USAFA males, voluntary SD significantly shifted the estimated decision weight away from the evidence and towards the base rate probability information, regardless of subjects' initial decision weights when NR. So, the directional effect of voluntary SD was to promote information weighting on Hard trials closer to that estimated for Easy trials. Qualitatively, this result is consistent with our previous work using a lab TSD protocol (Dickinson and Drummond, 2008). Thus, the effect whereby SD makes individuals deemphasize new information in decisions or evaluations may be present even when sleep restriction is voluntary and not extreme. An important implication of this result is that commonly experienced levels of sleep restriction may place one at a disadvantage if new information is particularly important. Consider the value of new evidence in a courtroom trial setting (Foreman, et al., 1997) or in medical diagnosis (Kononenko, 1993). Sleep deprived individuals may undervalue new information relative to its proper weighting, which may lead to an incorrect verdict or a suboptimal medical course of action. 
Voluntary sleep restriction of less than 6 hours nightly sleep over the course of a week did not significantly impact Bayesian accuracy on this task (i.e., they are just as likely to choose the Bayesian more likely box when SD as when NR). This is consistent with our previous study using a laboratory 24 hrs TSD protocol (Dickinson and Drummond, 2008). Nonetheless, the results suggest a reduced ability to fully incorporate multiple pieces of information into a decision during sleep restriction. Possibly the relative simplicity of the task (only two sources of information and a dichotomous choice) may not be powerful enough to detect accuracy differences. However, if changes reported here are amplified in more complex, real-world contexts, accuracy could well suffer (though that is an empirical question). Moreover, many real world decisions may not have a "right” answer (e.g., medical decisions) and a reduced ability to consider multiple sources of information could be detrimental for those decisions. Additional research using a more comprehensive design and/or complex stimuli is likely needed to answer some of these questions. Given how SD is estimated to alter the decision process, future studies may want to examine methods for training individuals to be aware of, and mitigate against, those alterations when sleep restricted. In that way, decisions could be made more consistently, regardless of sleep history.

\section{Limitations}

There are several limitations to this study. First, the simplistic task stimulus may limit our ability to generalize results to complex environments with more than two sources of information. Secondly, our study does not address the possibility of whether SD may paradoxically improve accuracy in specific environments, namely those where individuals fall prey to a "base rate fallacy” (Bar-Hillel, 1980; Kahneman and Tversky, 1973). This decision bias states base that rate information may be ignored if new evidence is available (i.e., underweighting of base rate data). It is therefore possible that in some instances a SD effect that 
shifts decision weights away from new evidence may lead to a more accurate choice than when normally-rested (see, e.g., our result for ASU male subjects on Hard trials in Fig. 4).

A final limitation of our study is that our results did not hold for USAFA male subjects, which somewhat confounds our ability to identify whether gender is truly mediating the SD effect (i.e., there is a clear gender effect on decision weights in Hard trials, but how SD impacts that effect changes simultaneously by gender and institution). Several differences exist between the USAFA and ASU data but it is not clear to what extent these differences may explain why voluntary SD impacted the decision process differentially for USAFA males. In the USAFA sample there was less variation in age among USAFA subjects, compensation was somewhat different, and diurnal preference was not a selection criteria. Young adults going to a military academy may also be fundamentally different from other young adults, perhaps in unobservable or unquantifiable ways. It was also the case that average sleep efficiency was significantly higher for the USAFA cadets than for ASU subjects (95.01 $\pm 5.11 \neq 82.15 \pm 6.49$, respectively: $\mathrm{p}<.01$ ). We consider that the ASU sample is likely a closer approximation to a general young adult sample than would be the USAFA sample for the subjects in the 18-25 year old age range-all our results hold if we re-estimate the ASU data on just the sub-sample of subjects between $18-25$ years of age ( $87 \%$ of the ASU sample). 


\section{TABLE 1: EASY TRIALS-Bayesian Choice Model Estimates.}

Dependent variable is subject Choice of $\operatorname{LEFT~BOX~}(=0,1)$. Estimations are random effects probit models, which cluster errors by subject. Subjects with sleep quantity between 6-7 hrs nightly are removed from the sample. Coefficient estimates are shown with standard errors in parentheses. ${ }^{* *}$, *** indicates significance at the .05 and .01 levels, respectively, for the two-tailed test. This table shows that voluntary SD does not impact the relative decision weights for EASY trials. $\mathrm{N}$ refers to total observations (i.e., subject*trials)

Note: for ASU male model, nightly sleep duration between 6-6.75 hr were removed instead of 6-7 hr due to failure of model to converge.

\begin{tabular}{|c|c|c|c|c|c|c|}
\hline Variable & $\begin{array}{c}\text { ASU } \\
\text { Pooled } \\
(\mathrm{n}=896)\end{array}$ & $\begin{array}{c}\text { ASU } \\
\text { Males } \\
(\mathrm{n}=448)\end{array}$ & $\begin{array}{c}\text { ASU } \\
\text { Females } \\
(\mathrm{n}=496)\end{array}$ & $\begin{array}{c}\text { USAFA } \\
\text { Pooled } \\
(\mathrm{n}=687)\end{array}$ & $\begin{array}{c}\text { USAFA } \\
\text { Males } \\
(\mathrm{n}=400)\end{array}$ & $\begin{array}{c}\text { USAFA } \\
\text { Females } \\
(\mathbf{n}=287)\end{array}$ \\
\hline Constant & $\begin{array}{c}.0379 \\
(.0824)\end{array}$ & $\begin{array}{c}.1466 \\
(.2873)\end{array}$ & $\begin{array}{c}.0142 \\
(.0864)\end{array}$ & $\begin{array}{l}.0575 \\
(.0724)\end{array}$ & $\begin{array}{l}-.1366 \\
(.0980)\end{array}$ & $\begin{array}{c}.0382 \\
(.1164)\end{array}$ \\
\hline Base Rate & $\begin{array}{c}1.3181 \\
(.1532)^{* * *}\end{array}$ & $\begin{array}{c}3.3273 \\
(1.6486)^{* *}\end{array}$ & $\begin{array}{c}1.2429 \\
(.1576)^{* * *}\end{array}$ & $\begin{array}{c}1.2313 \\
(.1327)^{* * *}\end{array}$ & $\begin{array}{c}1.3429 \\
(.1831)^{* * *}\end{array}$ & $\begin{array}{c}1.1180 \\
(.1984)^{* * *}\end{array}$ \\
\hline Evidence & $\begin{array}{c}.8542 \\
(.0655)^{* * *}\end{array}$ & $\begin{array}{c}1.9739 \\
(.7268)^{* * *}\end{array}$ & $\begin{array}{c}.7983 \\
(.0671)^{* * *}\end{array}$ & $\begin{array}{c}.6923 \\
(.0559)^{* * *}\end{array}$ & $\begin{array}{c}.7092 \\
(.0777)^{* * *}\end{array}$ & $\begin{array}{c}.6883 \\
(.0839)^{* * *}\end{array}$ \\
\hline SD & $\begin{array}{l}.0852 \\
(.1111)\end{array}$ & $\begin{array}{l}.0150 \\
(.3023)\end{array}$ & $\begin{array}{c}.0504 \\
(.1525)\end{array}$ & $\begin{array}{c}.0294 \\
(.1298)\end{array}$ & $\begin{array}{c}.0881 \\
(.1735)\end{array}$ & $\begin{array}{l}-.0374 \\
(.2107)\end{array}$ \\
\hline SD * Base Rate & $\begin{array}{l}.2397 \\
(.2153)\end{array}$ & $\begin{array}{l}-1.5941 \\
(1.6613)\end{array}$ & $\begin{array}{c}.0653 \\
(.2829)\end{array}$ & $\begin{array}{l}.0961 \\
(.2385)\end{array}$ & $\begin{array}{c}-.0202 \\
(.3190)\end{array}$ & $\begin{array}{c}.2274 \\
(.3659)\end{array}$ \\
\hline SD $*$ Evidence & $\begin{array}{l}-.0010 \\
(.0824)\end{array}$ & $\begin{array}{l}-1.0231 \\
(.7322)\end{array}$ & $\begin{array}{c}-.0979 \\
(.1191)\end{array}$ & $\begin{array}{c}.1925 \\
(.1025)\end{array}$ & $\begin{array}{c}.2241 \\
(.1376)\end{array}$ & $\begin{array}{l}.1328 \\
(.1564)\end{array}$ \\
\hline $\begin{array}{c}\text { Test of SD change in } \\
\text { relative coefficient weights } \\
\mathbf{H}_{\mathbf{0}}: \boldsymbol{\beta}\left(\mathrm{SD}^{*} \text { Evidence }\right)= \\
\boldsymbol{\beta}\left(\text { SD }^{*} \text { Base } \text { Rate }\right)\end{array}$ & $X^{2}=2.91$ & $X^{2}=0.37$ & $X^{2}=0.74$ & $X^{2}=.37$ & $X^{2}=1.33$ & $X^{2}=0.15$ \\
\hline Chi-squared (model) & $386.94 * * *$ & $162.47 * * *$ & $213.37 * * *$ & $285.36 * * *$ & $168.08 * * *$ & $111.43^{* * *}$ \\
\hline Log Likelihood & -328.7218 & -128.6499 & -197.5299 & -277.8267 & -154.3335 & -120.8469 \\
\hline
\end{tabular}




\section{TABLE 2: HARD TRIALS—Bayesian Choice Model Estimates.}

Dependent variable is subject Choice of $\operatorname{LEFT} B O X(=0,1)$. Estimations are random effects probit models, which cluster errors by subject. Subjects with sleep quantity between 6-7 hrs nightly are removed from the sample. Coefficient estimates are shown with standard errors in parentheses. **, *** indicates significance at the .05 and .01 levels, respectively, for the two-tailed test. This table shows that voluntary SD shifts the relative decision weights in Hard trials away from Evidence and toward Base Rates in three of the four samples (key coefficients to compare are shaded). $\mathrm{N}$ refers to total observations (i.e., subject*trials)

\begin{tabular}{|c|c|c|c|c|c|c|}
\hline Variable & $\begin{array}{c}\text { ASU } \\
\text { Pooled } \\
(\mathrm{n}=896)\end{array}$ & $\begin{array}{c}\text { ASU } \\
\text { Males } \\
(\mathrm{n}=400)\end{array}$ & $\begin{array}{c}\text { ASU } \\
\text { Females } \\
(\mathrm{n}=496)\end{array}$ & $\begin{array}{c}\text { USAFA } \\
\text { Pooled } \\
(\mathrm{n}=683)\end{array}$ & $\begin{array}{c}\text { USAFA } \\
\text { Males } \\
(\mathrm{n}=398)\end{array}$ & $\begin{array}{c}\text { USAFA } \\
\text { Females } \\
(\mathbf{n}=\mathbf{2 8 5})\end{array}$ \\
\hline Constant & $\begin{array}{c}.0577 \\
(.0658)\end{array}$ & $\begin{array}{c}.0998 \\
(.2635)\end{array}$ & $\begin{array}{c}.0570 \\
(.0702)\end{array}$ & $\begin{array}{c}.1053 \\
(.0645)\end{array}$ & $\begin{array}{c}.1389 \\
(.0885)\end{array}$ & $\begin{array}{c}.0623 \\
(.0974)\end{array}$ \\
\hline Base Rate & $\begin{array}{c}.7062 \\
(.1086)^{* * *}\end{array}$ & $\begin{array}{c}1.9211 \\
(.5057)^{* * *}\end{array}$ & $\begin{array}{c}.6399 \\
(.1170)^{* * *}\end{array}$ & $\begin{array}{c}.8575 \\
(1107)^{* * *}\end{array}$ & $\begin{array}{c}.8782 \\
(.1504)^{* * *}\end{array}$ & $\begin{array}{c}.8384 \\
(.1658)^{* * *}\end{array}$ \\
\hline Evidence & $\begin{array}{c}.9811 \\
(.1115)^{* * *}\end{array}$ & $\begin{array}{c}3.7177 \\
(.9507)^{* * *}\end{array}$ & $\begin{array}{c}.8479 \\
(.1183)^{* * *}\end{array}$ & $\begin{array}{c}.9589 \\
(.1090)^{* * *}\end{array}$ & $\begin{array}{c}.9389 \\
(.1470)^{* * *}\end{array}$ & $\begin{array}{c}.9922 \\
(.1649)^{* * *}\end{array}$ \\
\hline SD & $\begin{array}{c}.0942 \\
(.0913)\end{array}$ & $\begin{array}{c}.0623 \\
(.2754)\end{array}$ & $\begin{array}{c}.0834 \\
(.1438)\end{array}$ & $\begin{array}{c}-.1400 \\
(.1065)\end{array}$ & $\begin{array}{l}-.2207 \\
(.1442)\end{array}$ & $\begin{array}{l}-.0420 \\
(.1681)\end{array}$ \\
\hline SD * Base Rate & $\begin{array}{c}.5223 \\
(.1615)^{* * *}\end{array}$ & $\begin{array}{l}-.8520 \\
(.5221)\end{array}$ & $\begin{array}{c}1.2927 \\
(.3391)^{* * *}\end{array}$ & $\begin{array}{c}.1568 \\
(.1846)\end{array}$ & $\begin{array}{c}.1484 \\
(.2386)\end{array}$ & $\begin{array}{c}.2170 \\
(.2993)\end{array}$ \\
\hline SD * Evidence & $\begin{array}{c}.0605 \\
(.1566)\end{array}$ & $\begin{array}{c}-2.7662 \\
(.9577)^{* * *}\end{array}$ & $\begin{array}{c}.6474 \\
(.2881)^{* *}\end{array}$ & $\begin{array}{c}.1203 \\
(.1813)\end{array}$ & $\begin{array}{c}.3631 \\
(.2416)\end{array}$ & $\begin{array}{c}-.1622 \\
(.2868)\end{array}$ \\
\hline $\begin{array}{c}\text { Test of SD change in } \\
\text { relative coefficient weights } \\
\mathbf{H}_{\mathbf{0}}: \boldsymbol{\beta}\left(\mathrm{SD}^{*} \text { Evidence }\right)= \\
\boldsymbol{\beta}\left(\mathrm{SD}^{*} \text { Base } \text { Rate }\right)\end{array}$ & $\begin{array}{c}X^{2}= \\
33.94^{* * *}\end{array}$ & $\begin{array}{c}X^{2}= \\
13.27^{* * *}\end{array}$ & $\begin{array}{c}X^{2}= \\
23.88 * * *\end{array}$ & $\begin{array}{c}X^{2}= \\
.16\end{array}$ & $\begin{array}{l}X^{2}= \\
3.03\end{array}$ & $\begin{array}{c}X^{2}= \\
6.99 * * *\end{array}$ \\
\hline Chi-squared (model) & $188.22 * * *$ & $77.64^{* * *}$ & $90.27^{* * *}$ & $134.30^{* * *}$ & $87.18 * * *$ & $54.57 * * *$ \\
\hline Log Likelihood & -510.491 & -211.006 & -282.481 & -400.294 & -226.133 & -167.515 \\
\hline
\end{tabular}




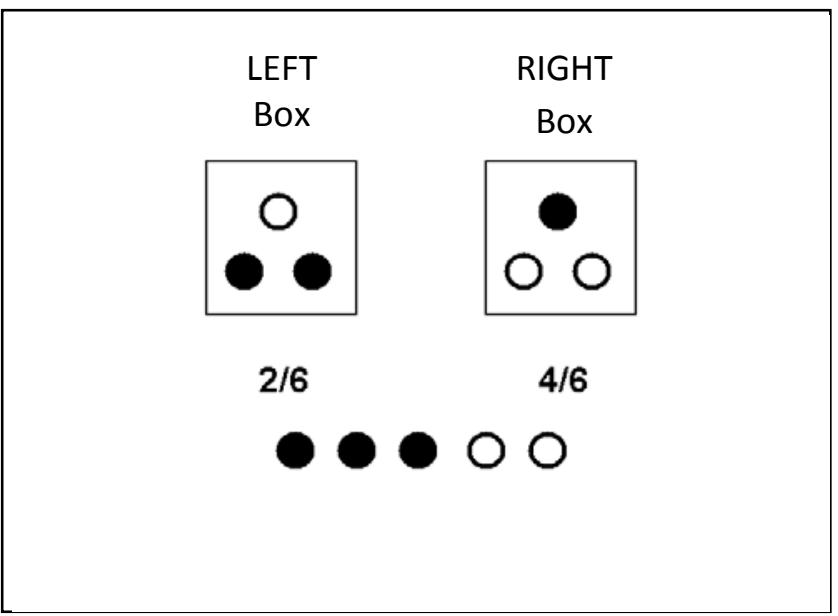

\section{FIGURE 1: Bayes' Rule (BR) task stimulus}

The task is to indicate from which Box balls were drawn. Subjects are shown the contents of each box, fractions below each Box indicate base rate odds for that Box, and the set of colored balls is the result of drawing five balls (with replacement) from the selected Box. There is no feedback on accuracy between trials. 


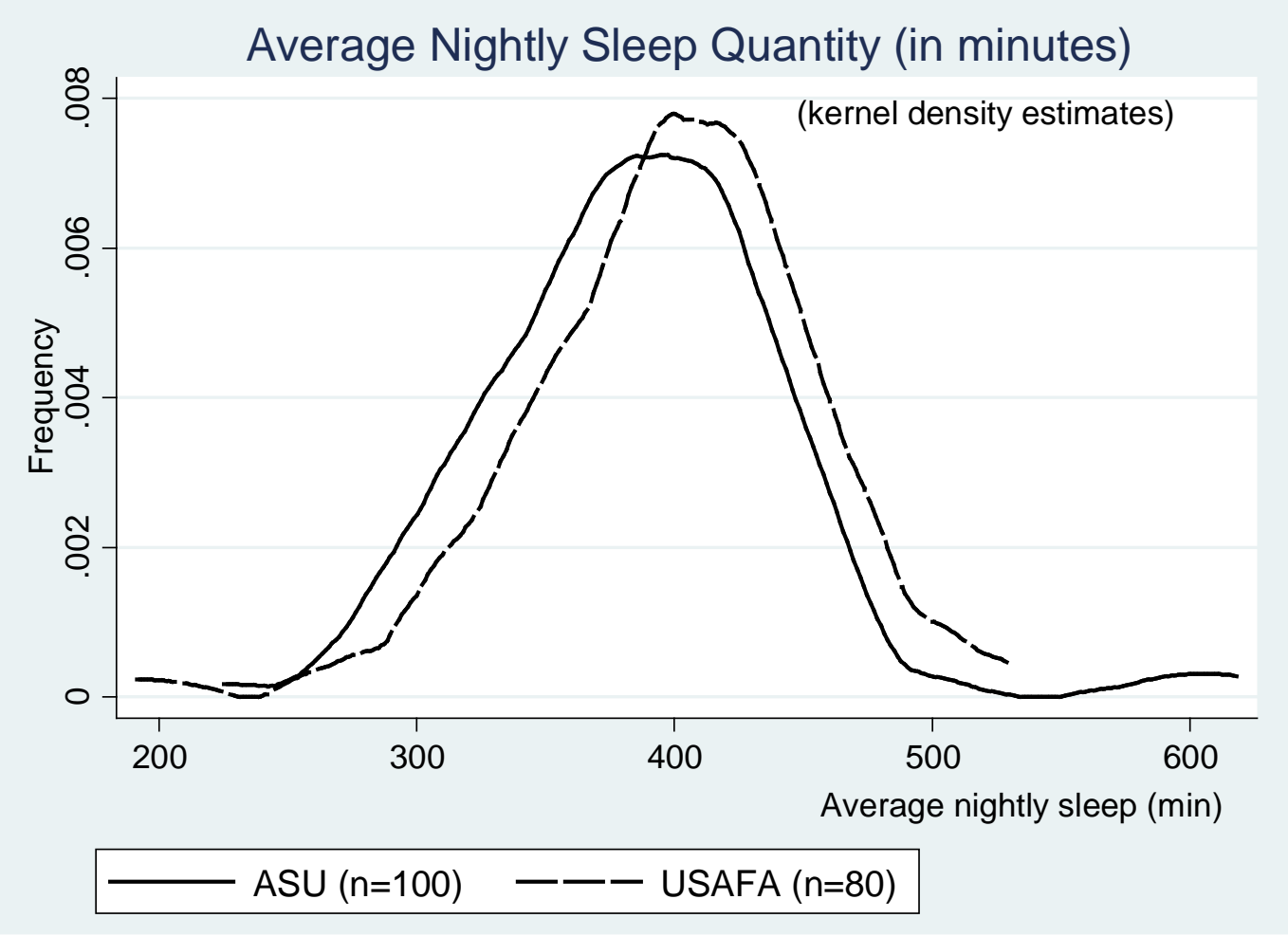

FIGURE 2: Frequency of ASU and USAFA Voluntary Sleep Choice Data

The kernel density non-parametrically estimates the probability density function of average nightly sleep using a standard Epanechnikov kernel and optimal smoothing bandwidth. The data showed that average nightly sleep at ASU (385.84 $\pm 57.5 \mathrm{~min})$ was significantly less than at USAFA (398.43 $\pm 54.90 \mathrm{~min})$ (Mann-Whitney test of means: p-value=.04). 


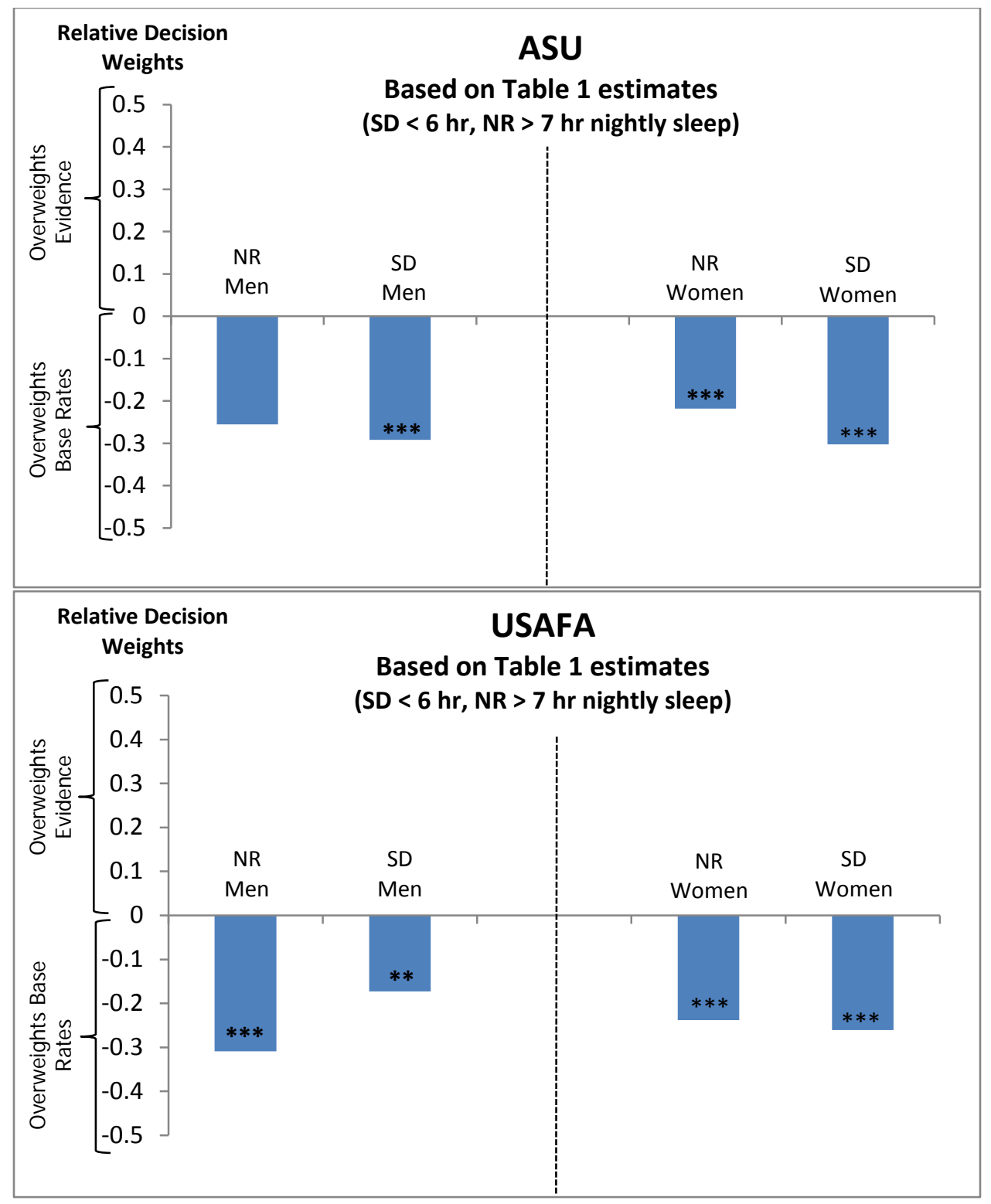

FIGURE 3: EASY TRIALS - Relative Decision Weights By Gender and Sleep Duration. Bars indicate relative decision weight differences between Evidence and Base Rate on the relative decision weight scale. ${ }^{* *}, * * *$ inside the bars indicate statistical significance at the .05 or .01 level, respectively, of the null hypothesis test of equal Evidence and Base Rate coefficients (based on Table 1 estimates). This figure visually displays the general overweighting of Base Rate on Easy trials, but the lack of significant differences in relative decisions weights estimated for the NR to SD subsamples. 

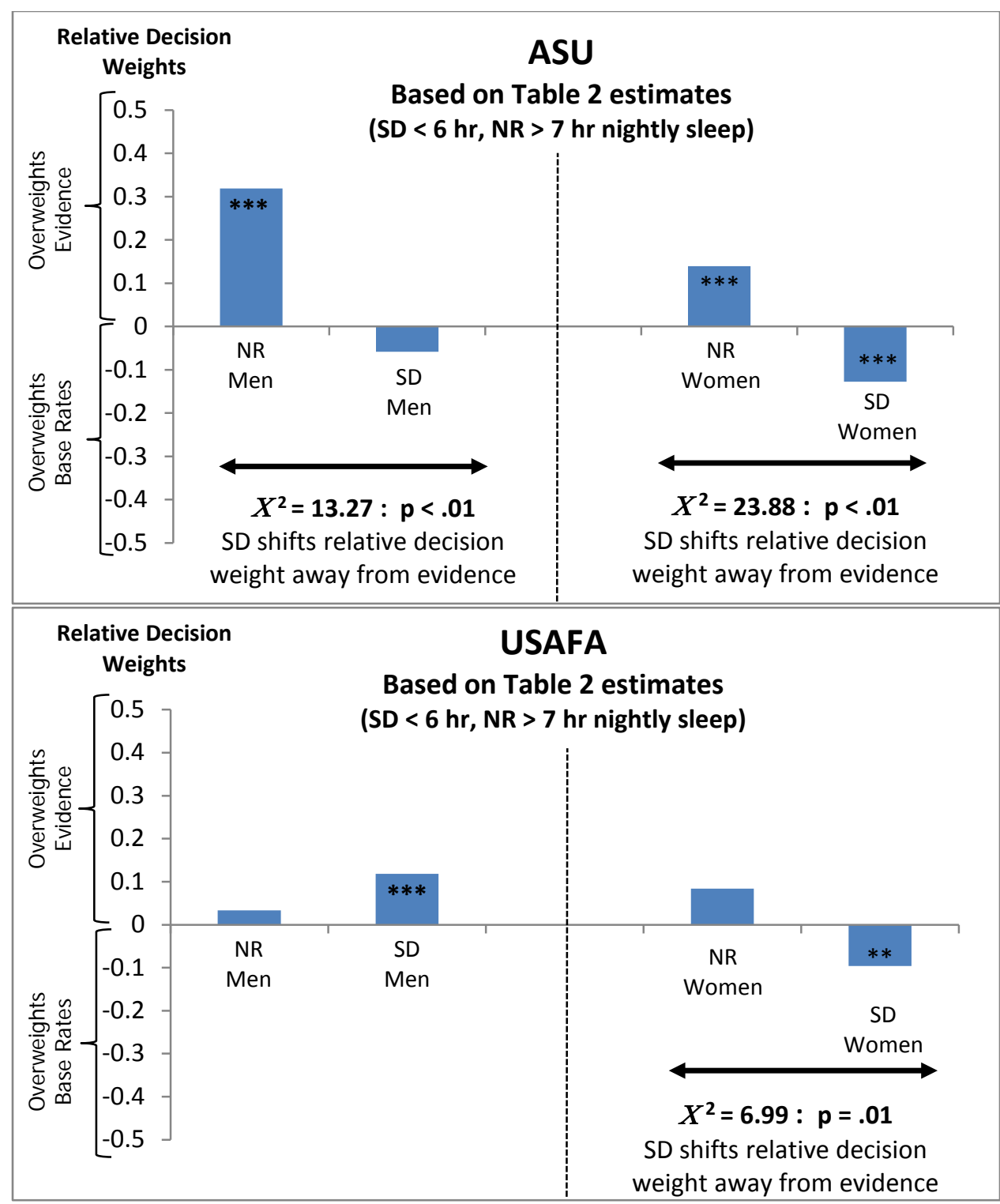

FIGURE 4: HARD TRIALS - Relative Decision Weights By Gender and Sleep Duration.

Bars indicate relative decision weight differences between Evidence and Base Rate on the relative decision weight scale. ${ }^{* *}, * * *$ inside the bars indicate statistical significance at the .05 or .01 level, respectively, of the test of the null hypothesis of equal Evidence and Base Rate coefficients (based on estimates from Table 2). This figure visually displays that subjects are more likely to weight (even overweight) Evidence compared to Base Rate on Hard trials. As noted in Table 2, the difference in the length of the bars comparing NR to SD subsamples indicates voluntary SD decreases relative decision weight on Evidence in 3 of the 4 samples. 


\section{ACKNOWLEDGEMENTS}

This work was supported by NSF grant \# SES-\#0727794 (to Dickinson), NSF grant \# SES0728504 (to Dyche), and NSF grant \#0729021 (to Drummond).

\section{REFERENCES}

Acheson, A., Richards, J. B., \& de Wit, H. (2007). Effects of sleep deprivation on impulsive behaviors in men and women. Physiology \& Behavior, 91, 579-587.

Adan, A., \& Almirall, H. (1991). Horne and Östberg morningness-eveningness questionnaire: a reduced scale. Personality and Individual Differences, 12, 241-253.

Allison, P. (1999). Comparing logit and probit coefficients across groups. Sociological Methods \& Research, 28, 186-208.

Bar-Hillel, M. (1980). The base-rate fallacy in probability judgments. Acta Psychologica, 44, 211-233.

Blacker, C. V. R., \& Mortimore, C. (1996). Randomized controlled trials and naturalistic data: time for a change? Human Psychopharmacology: Clinical and Experimental, 11, 353363.

Cameron, T.A. (2005). Updating subjective risks in the presence of conflicting information: An application to climate change. Journal of Risk and Uncertainty, 30, 63-97.

Chaumet, G., Taillard, J., Gagaspe, P., Pagani, M., Dinges, D. F., Pavy-le-Traon, . . Philip, P. (2009). Confinement and sleep deprivation effects on propensity to take risks. Aviation, Space, and Environmental Medicine, 80, 73-80.

Chelette, T.L, Albery, W.B., Esken, R.L. \& Tripp, L.D. (1998). Female exposure to high G: performance of simulated flight after 24 hours of sleep deprivation. Aviation Space and Environmental Medicine, 69, 862-8.

Dickinson, D. L., \& Drummond, S. P. A. (2008). The effects of total sleep deprivation on Bayesian updating. Judgment and Decision Making, 3, 181-190.

Foreman, L. A., Smith, A. F. M. \& Evett, I. W. (1997). Bayesian analysis of deoxyribonucleic acid profiling data in forensic identification applications (with discussion). Journal of the Royal Statistical Society, 160, 429-469.

Frey, D.J., Badia, P., \& Wright, K.P, Jr. (2004). Inter- and intra-individual variability in performance near the circadian nadir during sleep deprivation.” Journal of Sleep Research, 13, 305-315. 
Grether, D. (1980). Bayes' rule as a descriptive model: The representativeness heuristic. Quarterly Journal of Economics, 95, 537-557.

Harrison, Y., \& Horne, J. A. (2000). The impact of sleep deprivation on decision-making: A review. Journal of Experimental Psychology: Applied, 6, 236-249.

Horne, J. A. \& Östberg, O. (1976) A self-assessment questionnaire to determine morningnesseveningness in human circadian rhythms. International Journal of Chronobiology, 4, 97110.

Kahneman, D., \& Tversky, A. (1973). On the psychology of prediction. Psychological Review, 80, 237-251.

Killgore, W. D., Grugle, N. L., \& Balkin, T. J. (2012). Gambling when sleep deprived: Don’t bet on stimulants. Chronobioogy International, 29, 43-54.

Kononenko, I. (1993). Inductive and Bayesian learning in medical diagnosis. Applied Artificial Intelligence an International Journal, 7, 317-337.

Lundborg P, \& Andersson H. (2008). Gender, risk perceptions, and smoking behavior. Journal of Health Economics, 27, 1299-1311.

Lyvers, M., \& Tobias-Webb, J. (2010). Effects of acute alcohol consumption on executive cognitive functioning in naturalistic settings. Addictive Behaviors,35, 1021-1028.

McKenna, B. S., Dickinson, D. L., Orff, H., \& Drummond, S. P. A. (2007). The effects of one night of sleep deprivation on known-risk and ambiguous-risk decisions. Journal of Sleep Research, 16, 245-252.

McMenamin, T. M. (2007). A time to work: Recent trends in shift work and flexible schedules. Monthly Labor Review, Dec, 3-15.

National Sleep Foundation (2012). Sleep in America Polls. Retrieved from www.sleepfoundation.org.

Scholey, A. B., Benson, S., Neale, C., Owen, L., \& Tiplady, B. (2012). Neurocognitive and mood effects of alcohol in a naturalistic setting. Human Psychopharmacology: Clinical and Experimental, 27, 514-516.

Van Dongen, H. P. A., Maislin, G., Mullington, J. M., \& Dinges, D. F. (2003). The cumulative cost of additional wakefulness: Dose-response effects on neurobehavioral functions and sleep physiology from chronic sleep restriction and total sleep deprivation. Sleep, 26, 117-126. 
Venkatraman, V., Chuah, L. Y., Huettel, S. A. \& Chee, M. W. (2007). Sleep deprivation elevates expectation of gains and attenuates response to losses following risky decisions. Sleep, 2007, 30, 603-609.

Venkatraman, V., Heuttel, S. A., Chuah, L. Y., Payne, J. W., \& Chee, M. W. (2011). Sleep deprivation biases the neural mechanisms underlying economic preferences. The Journal of Neuroscience, 31, 3712-3718.

Volkow, N. D., Tomasi, D., Wang, G., Telang, F., Fowler, J. S., Logan, J., . . Ferré, S. (2012). Evidence that sleep deprivation downregulates dopamine D2R in ventral striatum in the human brain. Journal of Neuroscience, 32, 6711-6717.

Williams, R. (2009). Using heterogeneous choice models to compare logit and probit coefficients across groups. Sociological Methods \& Research, 37, 531-559. 